\title{
Recent Clinical Advances in the Management of Critically III Patients with Acute Renal Failure
}

\author{
William R. Clark ${ }^{a, b}$ Jeffrey J. Letteri ${ }^{b}$ Shigehiko Uchino ${ }^{c}$ Rinaldo Bellomo ${ }^{d}$ \\ Claudio Ronco ${ }^{\mathrm{e}}$ \\ ${ }^{a}$ Nephrology Division, Indiana University School of Medicine, Indianapolis, Ind., b Gambro Renal Products, \\ Lakewood, Colo., USA; ' Saitama Medical Center, Tokyo, Japan; ${ }^{\mathrm{d}}$ Austin Hospital, Heidelberg, Vic., Australia; \\ e Department of Nephrology, St. Bortolo Hospital, Vicenza, Italy
}

\section{Key Words}

Hemofiltration $\cdot$ Acute renal failure $\cdot$ Continuous renal

replacement therapy $\cdot$ RIFLE criteria

\begin{abstract}
Background: Significant progress has been made in the field of renal replacement therapy for critically ill patients with acute renal failure (ARF) over the past few years. This review highlights these developments. Methods: Recent studies assessing the clinical utility of the RIFLE classification system for the diagnosis of ARF were reviewed. Clinical outcome studies evaluating the effect of continuous renal replacement therapy (CRRT) dose and timing of initiation were assessed. The final review topic was the effect of dialysis modality on the recovery of renal function in ARF patients. Conclusions: Based on recent clinical studies, the increasing use of the RIFLE criteria is justified, as this approach appears to be a robust method for both the diagnosis of and prognostication in ARF. A large randomized trial involving convective CRRT supports the commonly used prescription of $35 \mathrm{ml} /$ $\mathrm{kg} / \mathrm{h}$ in clinical practice. Moreover, numerous recent outcome studies, also largely involving convective CRRT, provide a clinical rationale for the increasingly common clinical
\end{abstract}

practice of earlier initiation. Finally, several recent studies suggest CRRT, relative to conventional hemodialysis, results in a greater rate of renal recovery in ARF patients.

Copyright $\odot 2006$ S. Karger AG, Basel

\section{Introduction}

In recent years, a number of significant advances have occurred in the field of clinical acute renal failure (ARF) in the intensive care unit (ICU). One notable occurrence has been the development of a classification system designed to assist clinicians in the diagnosis of ARF. In addition, important new information about the dialytic management of ARF patients, especially in the field of continuous renal replacement therapy (CRRT), is available. These recent findings pertain to the influence of CRRT treatment dose and timing of treatment initiation on patient survival, and the effect of CRRT (vs. conventional hemodialysis) on recovery of renal function. Novel therapeutic approaches incorporating these new findings also have been described recently. The purpose of this review is to provide an overview of this new information and its potential impact on clinical practice.

\section{KARGER \\ Fax +41 613061234 E-Mail karger@karger.ch} www.karger.com
William R. Clark, MD

4322 Wythe Lane

Indianapolis, IN 46250 (USA)

Tel. +1 317691 1438, Fax +1 3178494599

E-MailWilliam.Clark@us.gambro.com 
Fig. 1. The RIFLE classification system for acute renal failure. Reprinted with permission from Kellum et al. [6].

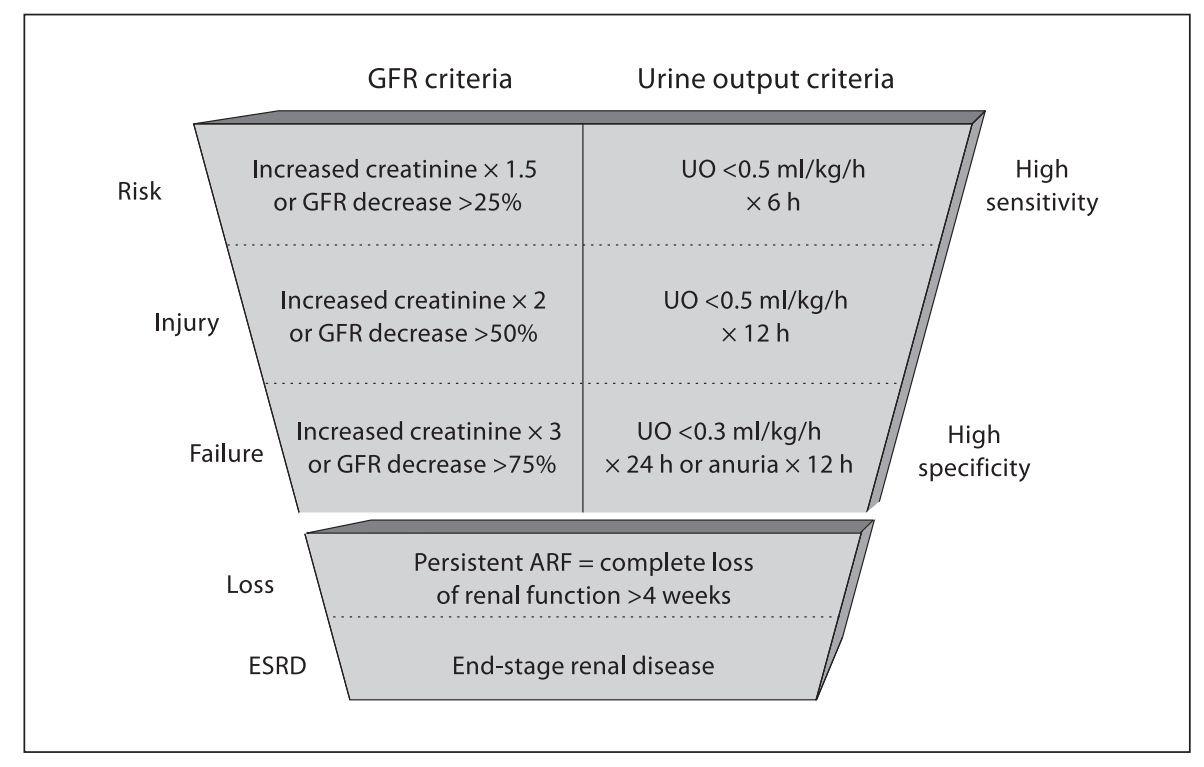

\section{The RIFLE Classification System for the Diagnosis of Acute Renal Failure}

Recent investigations have demonstrated that relatively small increases in the serum creatinine (Scr) level, even as small as $0.2 \mathrm{mg} / \mathrm{dl}$, have an adverse effect on ARF patient survival [1-4]. These results are a clear indication that Scr changes in hospitalized patients need to be assessed in a new light and demonstrate the need for methods permitting the more timely diagnosis of ARF. In this context, investigators affiliated with the Acute Dialysis Quality Initiative [5] have proposed a new approach (RIFLE) to defining and diagnosing ARF [6] (fig. 1). RIFLE is an acronym, for which the components are: $\mathrm{R}=$ risk of kidney injury; $\mathrm{I}=$ kidney injury; $\mathrm{F}=$ kidney failure; $\mathrm{L}=$ loss of kidney function, and $\mathrm{E}=$ endstage renal disease (ESRD). For each letter, there are two defining criteria: kidney function (as assessed by Scr) and urine output (as assessed by milliliters of urine per day, normalized to body weight). In the RIFLE approach, the worst of these two parameters (i.e., either the highest Scr or lowest daily urine output) is used for the assessment and compared to a patient's baseline (pre-ARF) value. Specifically, the first three RIFLE strata are as follows: (1) risk, glomerular filtration rate (GFR) decrease of $>25$ but $<50 \%$ or a urine output of $<0.5 \mathrm{ml} / \mathrm{kg} / \mathrm{h}$ for $6 \mathrm{~h}$; (2) injury, GFR decrease of $>50$ but $<75 \%$ or urine output of $<0.5 \mathrm{ml} / \mathrm{hg} / \mathrm{h}$ for $12 \mathrm{~h}$, and (3) failure, GFR decrease of $>75 \%$ or urine output of $<0.3 \mathrm{ml} / \mathrm{kg} / \mathrm{h}$ for $24 \mathrm{~h}$ or anuria for $>12 \mathrm{~h}$.
Bell et al. [7] performed the first study attempting to validate the RIFLE system with respect to its ability to predict mortality in critically ill ARF patients. In this retrospective study, researchers at the Karolinska Hospital in Stockholm assessed mortality over a 6-month period in 223 CRRT-treated patients during the period of 19952001. Overall, acuity of illness in the patient population was quite high, with $85 \%$ of the patients receiving mechanical ventilation and $78 \%$ receiving vasopressor support. Among numerous clinical factors that were potentially predictive of survival (including age and APACHE II score), only the RIFLE strata were statistically associated with mortality. Moreover, the RIFLE classification effectively stratified patients according to mortality risk, with a significant survival difference observed between patients with an ' $R$ ' or ' $I$ ' designation and patients with a 'F' or ' $\mathrm{L} / \mathrm{E}$ ' designation.

In another study, Abosaif et al. [8] applied the RIFLE classification system to 183 ARF patients admitted to a single ICU in the United Kingdom. Patients with a Scr of $>1.7 \mathrm{mg} / \mathrm{dl}$ on the first day of ICU admission were evaluated. In these patients, a baseline Scr was derived from the value 3 months before admission or, if this value was unavailable, the lowest value in the index hospitalization. Based on these two values, the Cockcroft-Gault equation was used to estimate the percentage change in GFR and classify patients into one of the RIFLE categories.

Overall ICU mortality in the entire cohort was $47.5 \%$. A significant association between RIFLE class and ICU mortality was observed, the latter being $38.3,50$, and 
$74.5 \%$ in the R, I, and F classes, respectively $(\mathrm{p}<0.001)$. Moreover, RIFLE class was found to be significantly correlated with both the raw APACHE II score and the APACHE II-related probability of death. Specifically, in the $\mathrm{R}, \mathrm{I}$, and $\mathrm{F}$ groups, the raw APACHE II scores (mean) were $20.9,22.2$, and 26.4 , respectively $(\mathrm{p}<0.001)$, while the probabilities of death (mean) were 31.4, 35.6, and $43.8 \%$, respectively $(\mathrm{p}<0.05)$. On the other hand, neither the raw Simplified Acute Physiology Score (SAPS) II nor the SAPS II-related probability of death differed significantly between the three groups. Nevertheless, based on receiver operator characteristic (ROC) curves, SAPS II tended to be a more sensitive indicator of mortality, at least in the $\mathrm{R}$ and I groups. The mean area under the curve (AUC) values for SAPS II were 0.78 and 0.76 in the $\mathrm{R}$ and I groups, respectively, while the same values for APACHE II were 0.62 and 0.72 , respectively. (An AUC value of 0.7 or greater demonstrates the utility of a particular variable as a risk predictor.) Neither SAPS II nor APACHE II was a significant discriminator in the $\mathrm{F}$ group.

A third recent study has also validated the clinical applicability of the RIFLE classification system. Kuitunen et al. [9] retrospectively evaluated 813 consecutive patients who had undergone cardiac surgery during 2003. For RIFLE classification, the highest concentration of plasma creatinine during a patient's stay in the ICU was used. The outcome variables in the study were 90 -day mortality and the need for prolonged ICU stay, defined as 5 days or more. For the entire population, these outcomes were 4.3 and $17.8 \%$, respectively, while $3.2 \%$ of patients required renal replacement therapy. However, outcome was strongly associated with RIFLE class, as 90-day mortality was $8,21.4$, and $32.5 \%$ in the R, I, and F classes, respectively ( $\mathrm{p}<0.0001)$. Likewise, the proportion of $\mathrm{pa}$ tients with an ICU stay of more than 5 days was $38.6,50$, and $70 \%$ in the R, I, and F classes, respectively ( $p<0.0001)$. RIFLE classification, change in plasma creatinine, and change in estimated GFR all were significant discriminators of 90-day mortality, having mean AUC values of $0.824,0.849$, and 0.829 , respectively, in ROC analyses. However, of these three variables, only RIFLE classification was found to be an independent risk factor for 90 day mortality in multivariate regression analysis.

In summary, the recent clinical outcome data demonstrating that even small increases in Scr are associated with increased mortality in ARF emphasize the critical importance of timely diagnosis. These three recent studies provide evidence the RIFLE classification system has clinical validity and suggest that clinicians can use it for both diagnosis and prognostication in ARF. Moreover, it is logical to assume that more timely diagnosis of ARF may lead to earlier therapeutic interventions, which may influence patient outcome (see below).

\section{Effect of CRRT Dose/Intensity on Outcome: New Treatment Approaches for the Management of ARF}

Early data from Storck et al. [10] suggested that the greater intensity of CRRT is associated with better patient outcome. In this study, patients were treated with either continuous arteriovenous hemofiltration $(\mathrm{CAVH})$ or continuous veno-venous hemofiltration $(\mathrm{CVVH})$ such that a wide range of ultrafiltration rates was obtained. Survival was found to be significantly higher in the CVVH group than in the CAVH group, in which the mean ultrafiltration rates were 15.5 and 7.5 liters/day, respectively. Whether the superior survival in the patients treated with CVVH rather than CAVH was related to the former's greater convective removal of small solutes or larger substances could not be determined from the data provided.

In a more recent, much larger prospective study performed by Ronco et al. [11], the effect of dose on outcome was assessed in 425 patients treated with CVVH. Patients were randomized to one of three groups based on dose, for which the surrogate was ultrafiltration rate normalized to body weight. The prescribed doses were 20,35 , and $45 \mathrm{ml} / \mathrm{h} / \mathrm{kg}$ while mean delivered ultrafiltrate volumes were 31,56, and 68 liters/day. Thus, at the time the study was performed, a conventional CRRT dose was prescribed to group 1 while the doses prescribed to groups 2 and 3 were significantly greater than those in typical clinical practice. CVVH was performed in the post-dilution mode with lactate-based substitution fluids and maximum blood flow rate of $240 \mathrm{ml} / \mathrm{min}$. There is a strong rationale for using ultrafiltration rate as a dose surrogate because a direct relationship exists between solute clearance and ultrafiltration rate in the post-dilution mode for molecules removed by filtration (i.e., non-adsorbed solutes) [12]. Although the benefit of convection is still present when hemofiltration is applied in the pre-dilution mode, the effect of dilution on replacement fluid requirements to achieve a certain solute clearance target needs to be considered [13].

Fourteen-day survival, the primary endpoint of the study, was significantly higher in groups 2 and 3 vs. group 1. In addition, hospital survival was significantly higher both in group $2(57 \%)$ and group $3(58 \%)$ vs. group 1 


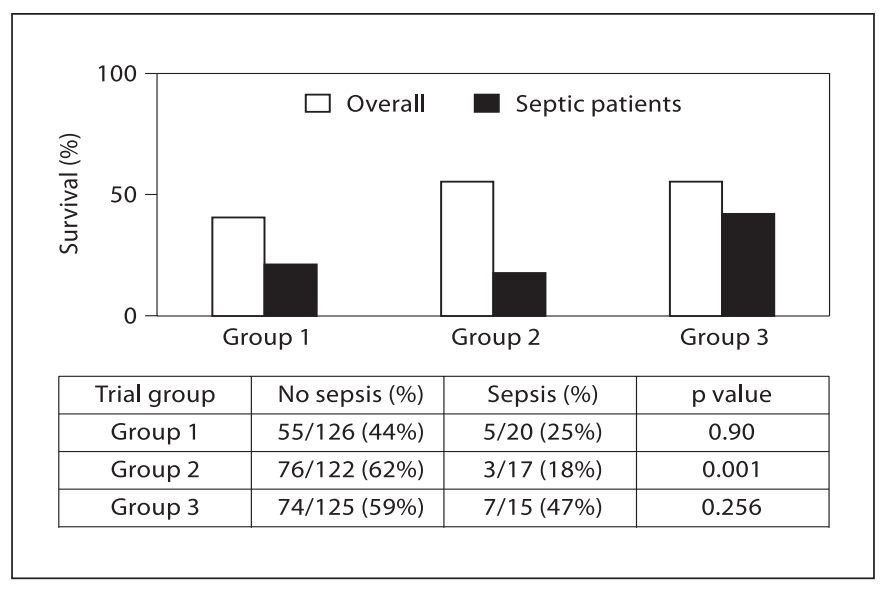

Fig. 2. Effect of CVVH dose of 35 (group 2) vs. $45 \mathrm{ml} / \mathrm{kg} / \mathrm{h}$ (group 3) on outcome of patients with sepsis-associated acute renal failure. Reprinted with permission from Ronco et al. [11].

(41\%). When all patients were considered, the difference in survival between groups 2 and 3 was not significant. However, in a sub-group analysis of patients with septic acute tubular necrosis, the outcome data suggested different clinical effects of 35 and $45 \mathrm{ml} / \mathrm{kg} / \mathrm{h}$ (fig. 2). In group 2, hospital survival in patients with septic acute tubular necrosis (18\%) was significantly lower than in all other patients (62\%). However, this same comparison in group 3 patients revealed no significant difference, as survival in the septic and non-septic groups was 47 and $59 \%$, respectively. It is important to note the septic subgroup was relatively small, accounting for only $11-14 \%$ of the total patient population.

In addition to the Ronco study, several other lines of clinical evidence suggest that the application of convective CRRT influences patient outcome favorably. Honore et al. [14] applied short-term, high-volume hemofiltration (STHVH) followed by conventional CVVH in 20 patients with refractory septic shock. The therapeutic regimen for STHVH consisted of an isovolemic 35-liter exchange over $4 \mathrm{~h}$, corresponding to a dose of $125 \mathrm{ml} / \mathrm{kg} / \mathrm{h}$ for a $70-\mathrm{kg}$ patient. Response to STHVH was defined by the following criteria: (1) $\geq 50 \%$ in cardiac index within $2 \mathrm{~h}$; (2) $\geq 25 \%$ in mixed venous saturation within $2 \mathrm{~h}$; (3) increase in the arterial $\mathrm{pH}$ to $>7.3$ within $4 \mathrm{~h}$, and (4) $\geq 50 \%$ reduction in epinephrine dose within $4 \mathrm{~h}$. A hemodynamic 'response' was defined as the attainment of all four of these criteria by a given patient. A positive response was associated with survival at 28 days in 9 of 11 patients while all 9 non-responding patients were dead at 28 days. Furthermore, the delay time, defined as the time from admission to the ICU to the initiation of STHVH, was significantly shorter in responders than in non-responders (mean 6.5 vs. $13.7 \mathrm{~h}, \mathrm{p}<0.01$ ).

In an analogous study, Piccinni et al. [15] evaluated the effect of early isovolemic hemofiltration (EIHF) in 80 oliguric patients with septic shock. All patients had at least two systemic inflammatory response syndrome criteria [16], a clinically identified focus of infection, the combination of high cardiac output and low systemic vascular resistance, and the presence of both acute renal injury and acute lung injury. In 40 patients, EIHF at a dose of $45 \mathrm{ml} / \mathrm{kg} / \mathrm{h}$ was applied within $12 \mathrm{~h}$ of ICU admission for $6 \mathrm{~h}$. These patients subsequently received conventional CVVH $(20 \mathrm{ml} / \mathrm{kg} / \mathrm{h})$ for a minimum of 3 days. Treatment dose in the control group $(20 \mathrm{ml} / \mathrm{kg} / \mathrm{h})$ reflected standard clinical practice prior to the use of EIHF. Moreover, classic ARF criteria were used to guide initiation of therapy in the control group [17].

The primary endpoint was improvement in pulmonary function defined by the $\mathrm{PaO}_{2} / \mathrm{FiO}_{2}$ ratio at $48 \mathrm{~h}$, which was significantly higher in the EIHF group than the control group (mean 240 vs. $160 \mathrm{~mm} \mathrm{Hg;} \mathrm{p} \mathrm{<} \mathrm{0.05).}$ Relative to the conventional group, other hemodynamic parameters that were significantly different in the EIHF group included lower heart rate, higher mean arterial pressure, higher systemic vascular resistance, and lower noradrenaline dose. Moreover, essentially all of the clinical outcome parameters that were measured also differed between the 2 groups (table 1). With respect to ventilator dependence, a significantly higher percentage of EIHF patients than conventionally treated patients were successfully weaned (70 vs. $37 \%$, respectively; $p<0.05)$ while the duration of mechanical ventilation was also significantly shorter (mean 11 vs. 20 days; $p<0.05$ ). Furthermore, both ICU stay (mean 12 vs. 16 days; $\mathrm{p}<0.05$ ) and hospital stay (mean 19 vs. 34 days; $p<0.05$ ) were significantly shorter in the EIHF vs. the conventional group. Finally, predicted 28-day survival based on Kaplan-Meier estimates was $55 \%$ in the EIHF group and $27.5 \%$ in the conventional group $(p=0.005)$. It is worthwhile to note that mortality in the conventional group (approximately $70 \%$ ) is very consistent with the literature [18-24], while the $45 \%$ mortality in the EIHF group represents a substantial improvement in outcome.

Another study supporting the application of early convective CRRT in refractory septic shock was recently reported by Page et al. [25]. Based on accepted clinical definitions for sepsis, refractory circulatory failure, acute renal injury, and acute lung injury, patients having all of these characteristics were enrolled in the study. A stan- 
Table 1. Effect of early isovolemic hemofiltration (EIHF) on outcome in patients with septic shock

\begin{tabular}{|c|c|c|c|}
\hline & $\begin{array}{l}\text { EIHF } \\
(n=40)\end{array}$ & $\begin{array}{l}\text { Control } \\
(\mathrm{n}=40)\end{array}$ & $\mathrm{p}$ \\
\hline Successful weaning, $\mathrm{n}$ & $28(70 \%)$ & $15(37 \%)$ & $<0.001$ \\
\hline Duration of MV, days & $11 \pm 3$ & $20 \pm 5$ & $<0.001$ \\
\hline Independence from vasopressor support, $\mathrm{n}$ & $30(75 \%)$ & $10(25 \%)$ & $<0.001$ \\
\hline ICU stay, days & $12 \pm 5$ & $16 \pm 4$ & 0.002 \\
\hline Hospital stay, days & $19 \pm 5$ & $34 \pm 4$ & $<0.001$ \\
\hline ICU survival, $\mathrm{n}$ & $28(70 \%)$ & $16(40 \%)$ & 0.003 \\
\hline $\begin{array}{l}\text { Predicted survival based on individual risk } \\
\text { of death }\end{array}$ & $41 \pm 12$ & $40 \pm 10$ & n.s. \\
\hline 28-day survival, $\mathrm{n}$ & $22(55 \%)$ & $11(27.5 \%)$ & 0.005 \\
\hline $\begin{array}{l}\text { Predicted survival based on individual risk } \\
\text { of death }\end{array}$ & $41 \pm 12$ & $40 \pm 10$ & n.s. \\
\hline
\end{tabular}

Reprinted with permission from Piccinni et al. [15]. dardized resuscitation protocol, including specific agents for volume, inotropic, and vasopressor support, was used. After a maximum 12-hour resuscitation period, in the case of a worsening metabolic acidosis, continuous venovenous hemodiafiltration (CVVHDF; ultrafiltration rate, 2 liters/h; dialysate flow rate, 1 liter/h) was initiated.

A total of 60 patients comprised this study. Similar to the approach used in the Honore et al. study [14], patients were categorized as responders or non-responders based on the effect of CVVHDF therapy on metabolic acidosis (as represented by base excess). In the responder group, base excess increased from $-11 \mathrm{mmol} / \mathrm{l}$ immediately after the resuscitation period (baseline) to approximately $-5 \mathrm{mmol} / \mathrm{l}$ after $24 \mathrm{~h}$ of CVVHDF. On the other hand, base excess continued to fall from the baseline mean value of $-13 \mathrm{mmol} / \mathrm{l}$ in the non-responder group. Based on measurements at the same time points, catecholamine requirements decreased significantly and urine output increased significantly in the responder group while no significant changes were observed in the non-responder group. Finally, overall unadjusted hospital mortality was $53 \%$ but differed significantly in the two groups. In the responder group, the predicted mortality based on the SAPS [26] was $67 \%$ while the actual mortality was $30 \%$. On the other hand, all patients in the non-responder group died ( $100 \%$ mortality; $\mathrm{p}<0.0001$ vs. actual mortality in responder group), which was actually somewhat higher than the predicted mortality (87\%).

The four preceding clinical studies all suggest that high-dose convective therapy improves survival in the most critically ill ARF patients (i.e., the severe sepsis/septic shock sub-groups), especially when applied early. Based on these studies and taking into account the dis- parate clinical results achieved in the past with high-volume hemofiltration [27-31], the Vicenza group has proposed a novel convective approach for the management of septic shock termed pulse high-volume hemofiltration $[32,33]$. These investigators observed correctly that previous pharmaceutical interventions targeting a specific inflammatory mediator and administered at a discrete time point have largely failed because of the dynamic nature of the septic process $[34,35]$, the exception being activated protein C [36]. On the other hand, the ability of CRRT to modulate both pro-inflammatory and anti-inflammatory mediators, even to a small extent, over a sustained period of time may be a significant attribute. Moreover, the biological effects of inflammatory mediator modulation may not be reflected by changes in plasma concentrations but instead occur at a tissue level.

In pulse high-volume hemofiltration, a balance is struck between biological plausibility and clinical practicality. The regimen consists of a brief 'burst' of hemofiltration $(85 \mathrm{ml} / \mathrm{kg} / \mathrm{h}$ for $6-8 \mathrm{~h})$ followed by a relatively standard dose of $35 \mathrm{ml} / \mathrm{kg} / \mathrm{h}$ for the remaining 16-18 h. On a time-averaged basis, this corresponds to a dose of approximately $48 \mathrm{ml} / \mathrm{kg} / \mathrm{h}$. The foundation of the biological rationale for this approach lies in the multi-pool kinetics of inflammatory mediators [37]. The initial therapy pulse makes possible the convective removal of inflammatory mediators from the plasma compartment while having little effect on other body compartments. During the standard dose phase, the plasma compartment can then 'refill' over several hours in preparation for the next pulse period [38] in a manner analogous to apheresis techniques [39]. Moreover, the extended standard dose phase allows for homeostasis to be achieved. 
The practicality of this approach lies in the need to address the logistics of providing high fluid exchange rates for only a relatively brief period of time each day. The $\mathrm{Vi}$ cenza group has recently reported its initial clinical experience with this technique in a group of 15 patients with severe sepsis [33].

A single-center study from the Netherlands attempted not only to corroborate the dose findings of the Ronco trial but also to assess the effect of timing of CRRT initiation on patient survival [40]. In this study performed by Bouman et al. [40], 106 patients were randomized to one of three groups: early high volume hemofiltration (EHV), early low volume hemofiltration, and late low volume hemofiltration. As in the Ronco trial [11], post-dilution hemofiltration was performed exclusively. Despite a minimum ultrafiltration volume of 72 liters/day in the early high volume hemofiltration group, the blood flow rate prescribed was only $200 \mathrm{ml} / \mathrm{min}$. Based on a standard hematocrit of $30 \%$, this combination implies a minimum filtration fraction of $36 \%$ in this group. This may be the explanation for the relatively high frequency of filter changes in the early high volume hemofiltration group (mean duration of use, $13.6 \mathrm{~h}$ ) and raises questions about actual dose delivered in this study. Neither treatment dose nor timing of treatment intervention had a significant effect on 28-day survival, which was significantly higher $(75-80 \%$ in all three groups) than has been reported routinely over the past several years for the critically ill ARF population [18-24].

This study has a number of shortcomings that require further comment. First, it should be emphasized that the size of the patient population in this trial was only $25 \%$ of that in the Ronco trial. Thus, the issue of whether or not the study had sufficient statistical power is relevant. Second, valid questions about the nature of the patient population can be raised. As noted above, the exceedingly low mortality in this study $(20-25 \%$ at 28 days) differs substantially from the typical $50-60 \%$ value characteristic of the critically ill ARF population. In contrast, 28-day mortality in the low-dose group of the Ronco trial was in this expected range. For the overall patient population in the Bouman study, another feature uncharacteristic of the critically ill ARF patient population was the exceedingly low $(0.9 \%)$ failure rate for recovery of renal function. On the other hand, several recent studies have suggested that the rate of non-recovery of renal function is minimally $10 \%$ and may be as high as $35 \%$ of hospital survivors [41-44] (see below). These survival and recovery of renal function data suggest that the Bouman study population was not representative of the critically ill ARF population. Thus, the results may be difficult to apply in clinical practice.

It is important to emphasize that the relationship between solute clearance and flow rate in diffusive therapies differs significantly from that in convective therapies. Based on mass transfer considerations, the expected clearance of small solutes during CVVHD and post-dilution CVVH is the same [45]. However, as the solute molecular weight increases, the relevance of diffusion diminishes and the benefits of convection become increasingly apparent [46]. For acute dialysis modalities, these principles have been substantiated in both modeling and clinical studies. Liao et al. [47] performed a kinetic comparison of intermittent hemodialysis (daily 4-hour treatments), sustained low-efficiency dialysis (SLED; daily 12hour treatments), and pre-dilution CVVH (ultrafiltration rate, $35 \mathrm{ml} / \mathrm{kg} / \mathrm{h}$ ) These investigators employed the equivalent renal clearance concept [48] to compare effective solute removal for these modalities. Their analyses indicated similar effective urea clearances for $\mathrm{CVVH}$ and SLED of 33 and $31 \mathrm{ml} / \mathrm{min}$, respectively, both of which were substantially higher than that delivered by daily intermittent hemodialysis $(21 \mathrm{ml} / \mathrm{min})$. On the other hand, the estimated $\beta_{2}$-microglobulin $\left(\beta_{2} \mathrm{M}\right)$ clearances for CVVH and SLED were 18 and $4 \mathrm{ml} / \mathrm{min}$, respectively. Daily intermittent hemodialysis with a high-flux dialyzer was estimated to provide an intermediate $\beta_{2} \mathrm{M}$ clearance of $7 \mathrm{ml} / \mathrm{min}$.

These modeled data are extended by a recent clinical study in which effluent collections were used to quantify solute removal in pre-dilution CVVH (2.5 liters/h) and a SLED system utilizing a high-flux filter [49]. (Consistent with clinical practice in the US, the above modeling study employed a low-flux dialyzer.) Indeed, Kielstein et al. [49] corroborated Liao's findings by demonstrating that urea and creatinine removal during CVVH and SLED are similar. However, $\beta_{2} \mathrm{M}$ removal was twofold greater in CVVH vs. SLED, even with the use of a high-flux filter in the latter therapy.

A more recent study performed by Ricci et al. [50] reinforces the importance of convection in achieving solute clearance over a broad molecular weight spectrum in CRRT. Based on a common prescription of $35 \mathrm{ml} / \mathrm{kg} / \mathrm{h}$ effluent flow rate, these investigators measured clearance of urea, creatinine, and $\beta_{2} \mathrm{M}$ during CVVH and CVVHD in a cross-over study. The median urea (31.6 vs. $35.7 \mathrm{ml} /$ $\mathrm{min}$ ) and creatinine ( $38.1 \mathrm{vs.} 35.6 \mathrm{ml} / \mathrm{min}$ ) in CVVH and CVVHD, respectively, were similar. However, median $\beta_{2} \mathrm{M}$ clearance in $\mathrm{CVVH}$ was higher than that in CVVHD (16.3 vs. $6.3 \mathrm{ml} / \mathrm{min}$, respectively; $\mathrm{p}=0.055)$. It 
must be emphasized this borderline statistically significant difference was observed despite the fact this trial was markedly underpowered.

\section{Timing of Treatment Initiation in CRRT}

In addition to supporting the importance of convective CRRT, recent data also suggest early initiation of CRRT improves patient outcome. As discussed above, studies performed by Honore et al. [14], Piccinni et al. [15], and Page et al. [25] indicate that relatively early application of hemofiltration in patients with septic shock is clinically beneficial. A relevant question is whether the survival advantage in these studies was mediated by enhanced convective solute removal, early initiation, or a combination of both factors. The studies were not designed to address this question. Nevertheless, a growing body of evidence indicates that timing of treatment initiation plays an important role in patients treated with CRRT. It is important to emphasize that all of the studies suggesting that early initiation is clinically beneficial employed convection-based CRRT.

In the Ronco dose trial [11], the mean blood urea nitrogen (BUN) at the time of CVVH initiation was significantly lower among survivors vs. non-survivors within each dose group. Based on the assumption that BUN is a reasonable surrogate for timing of treatment initiation, this finding indicates that early initiation of CRRT has a significant effect on survival that is independent of therapy dose. A reasonable challenge to this conclusion is that a high BUN at treatment initiation is an indicator of a very ill patient with marked hypercatabolism [51-53]. However, in this analysis, no clinical differences at baseline were observed between the surviving and non-surviving patients. Furthermore, timing of treatment initiation has been shown to be a significant predictor of outcome in several other studies.

Gettings et al. [54] retrospectively analyzed the effect of timing of CRRT initiation on outcome in 100 posttraumatic ARF patients. These investigators designated patients with $B U N$ values of $<60 \mathrm{mg} / \mathrm{dl}$ at the time of CVVHDF initiation as 'early starters' and patients with BUN values of $>60 \mathrm{mg} / \mathrm{dl}$ as 'late starters'. No significant differences in clinical parameters, including illness severity score, percentage of patients in shock, BUN, and arterial lactate concentration, between the two groups existed at the time of hospital admission. At the time of CRRT initiation, the mean BUN in the early and late start groups was 43 and $95 \mathrm{mg} / \mathrm{dl}$, respectively ( $\mathrm{p}<0.0001)$, and these values occurred on average 10 and 19 days after hospital admission, respectively ( $\mathrm{p}<0.0001)$. Furthermore, serum albumin was significantly lower in the late start group than the early start group $(2.50$ vs. $2.61 \mathrm{~g} / \mathrm{dl}$; p < $0.05)$, a finding possibly related to a greater degree of either volume overload or pro-inflammation in the former group. The CRRT prescription did not differ between the two groups, as the mean ultrafiltrate volume was approximately 12 liters/day and the mean dialysate flow rate was approximately 1.1 liters/h for both. Moreover, these BUN values could not be explained by differences in nitrogen administration, as both groups received approximately $140 \mathrm{~g}$ (mean) of dietary protein on a daily basis.

For the first 3 days of CRRT, the mean BUN was significantly higher in the late start group but did not differ significantly from the mean BUN in the early start group from day 4 onward. In fact, the azotemia profiles for the two groups closely approximated one another during this later time period, staying within a relatively narrow BUN band of $65-70 \mathrm{mg} / \mathrm{dl}$ in a quasi-steady state. As the steady state $\mathrm{BUN}$ is related to the ratio of protein catabolic rate to the treatment dose [55] and because the CRRT prescription did not differ between the two groups, this finding suggests the degree of hypercatabolism also did not differ significantly between the two groups. Nevertheless, although this rough estimate of overall illness severity did not suggest a clinical difference between the two groups, hospital survival was significantly higher in the early start group than in the late start group (39 vs. $28 \%$; $\mathrm{p}<0.05$ ). As discussed above, both of these figures (i.e., hospital mortality of approximately $60-70 \%$ ) are consistent with the consensus in the literature, suggesting that a representative patient population was studied.

Additional studies suggesting that early application of CRRT improves patient outcome have been published more recently. In a single-center trial, Bent et al. [56] assessed the effect of relatively early intervention with CVVH in 65 patients with ARF after cardiac surgery. The patient population was characterized by advanced age (mean 71 years), significant preexisting chronic kidney disease ( $45 \%$ of patients), and a high degree of oliguria (79\% of patients) and intra-aortic balloon pump dependence $(32 \%)$ in the postoperative period. The CVVH prescription included a standard ultrafiltration rate of 2 liters/ $\mathrm{h}$ while the mean time between operation and $\mathrm{CVVH}$ initiation was 2.4 days. The predicted mean death rate in this population, based on the Liano ARF model [57], was $66 \%$. However, the observed death rate was only $40 \%$ ( $p<0.005$ vs. predicted). The authors attributed this improved survival not only to early intervention but also to 
the relatively high dose of therapy delivered (at least for that time period).

Elahi et al. [58] performed another study assessing the potential benefit of early CRRT initiation in the cardiac surgery population. Of the 1,264 patients who underwent cardiac surgery at their institution over a 1-year period, the 64 patients ( $5 \%$ of the total) who required postoperative renal replacement therapy for ARF were evaluated. These patients were divided into two categories. Group I (late hemofiltration) consisted of patients for whom CVVH was initiated based on the following criteria: BUN $\geq 84 \mathrm{mg} / \mathrm{dl}$; creatinine $\geq 2.8 \mathrm{mg} / \mathrm{dl}$; or serum potassium $\geq 6 \mathrm{mmol} / \mathrm{l}$. These criteria were applied regardless of the urine output. In group II (early hemofiltration) CVVH was initiated when the urine output was less than $100 \mathrm{ml}$ during a consecutive 8-hour period, regardless of the biochemical parameters. At baseline, the ARF population was characterized by advanced age (mean 70 years) along with a high prevalence of both heart failure (56\% either NYHA class 3 or 4 ) and chronic kidney disease (mean serum creatinine $1.8 \mathrm{mg} / \mathrm{dl}$ ).

In the early hemofiltration group, the mean time between surgery and CVVH initiation was approximately $18 \mathrm{~h}$ while the same parameter in group I was 2.55 days $(\mathrm{p}<0.001)$. This time difference was associated with a significantly lower BUN in the early than the late group (67 vs. $75 \mathrm{mg} / \mathrm{dl} ; \mathrm{p}<0.05$ ). It is important to note that early CVVH initiation did not lead to overall prolongation of therapy, as the mean treatment duration (4.6 days) was identical in the two groups. The hospital mortality for the entire ARF group was 31\%. However, mortality was significantly lower in the early start group than in the late start group ( 22 vs. $43 \%$; $\mathrm{p}<0.05$ ). Moreover, mean ICU stay was significantly reduced in the early start group relative to the late start group ( 8.5 vs. 12.5 days, $\mathrm{p}<0.05)$, as was mean hospital stay (15.4 vs. 20.9 days; $\mathrm{p}<0.05$ ). Finally, the percentage of patients developing multi-organ failure after CVVH initiation was significantly lower in the early start vs. late start group ( 19 vs. $29 \%$; $\mathrm{p}=$ 0.01 ).

Sugahara et al. [59] employed urine output as a CRRT initiation criterion in a series of patients with ARF after cardiac surgery. Patients were enrolled in the study when urine output fell below $30 \mathrm{ml} / \mathrm{h}$ and the serum creatinine increased at a rate of $0.5 \mathrm{mg} / \mathrm{dl} / \mathrm{day}$ or more. The early start group was comprised of patients for whom CRRT was initiated when urine output was $<30 \mathrm{ml} / \mathrm{h}$ for three consecutive hours (daily urine output approximately $<750 \mathrm{ml}$ ). On the other hand, CRRT was initiated in the conventional start group when urine output was
$<20 \mathrm{ml} / \mathrm{h}$ for two consecutive hours (daily urine output approximately $<500 \mathrm{ml}$ ). At baseline, the two groups were well matched with respect to age, presence of underlying comorbidities (diabetes, hypertension, hypercholesterolemia), serum creatinine, and APACHE II score (mean 19 vs. 18 in the early and conventional groups, respectively). Mean urine output was preserved for the first several days in the early start group and began to increase on day 6 of CRRT, with the difference between baseline becoming significant on day 8 . On the other hand, the mean urine output decreased during the first 3 days of CRRT in the conventional start group and, although it increased subsequently, the change from baseline was never significant over the 14-day period of evaluation. Moreover, survival at 14 days was significantly higher in the early start group vs. the conventional start group.

Finally, the primary purpose of a large multicenter cohort study recently performed by Mehta et al. [60] was to explore the potential association between diuretic use in $\mathrm{ARF}$ and outcome. However, at least inferential information about the possible effect of the timing of dialysis initiation on outcome also was provided. In this study, the effect of diuretic use in 552 ARF patients on all-cause hospital mortality and non-recovery of renal function was assessed. Relative to patients not treated with diuretics on the first day of nephrology consultation in the ICU, patients receiving diuretics had a significantly lower mean BUN, serum creatinine, and cardiac index and a significantly higher presence of cardiopulmonary failure. After adjustment for covariate differences based on regression analysis, patients receiving diuretics had a $77 \%$ higher risk of hospital mortality or non-recovery of renal function relative to patients not treated with diuretics. Moreover, patients receiving relatively high-dose loop diuretics, which had no significant impact on urine output, had the highest risk of death. Specifically, within 1 week of nephrology consultation in the ICU, essentially all patients receiving $\geq 1 \mathrm{mg}$ furosemide $/ \mathrm{ml}$ of urine output on a daily basis had died. The authors suggested the explanation for these data is the delay in dialysis initiation while healthcare providers await a potential response to diuretics, at least in some patients. Based on these data, patients with a declining urine output despite high doses of loop diuretics might be particularly susceptible to the deleterious effects of delayed initiation of dialysis. Thus, although the potential detrimental effects of diuretic use in ARF has not been confirmed in a second recent study [61], the Mehta et al. study [60] provides further suggestive evidence that timely initiation of dialysis is an important clinical consideration. 


\section{Effect of Dialysis Modality on Recovery of Renal Function in ARF}

Except for patient survival, recovery of renal function is the most important clinical goal in patients with ARF. In spite of this and the long-term clinical and economic implications of the failure to recover renal function, this issue has received relatively little attention in the past. The largest study addressing this issue evaluated 1,094 patients with severe ARF between 1984 and 1995 [62]. (Severe ARF was defined as an acute uremic emergency with a serum creatinine of $\geq 600 \mu \mathrm{mol} / \mathrm{l}$ and/or the need for dialysis.) Of patients surviving to 90 days, $16.7 \%$ were still dependent on dialysis at that time. The major factors contributing to ARF were: parenchymal disease, $42 \%$; medical/surgical, 23\%; vascular (including cardiac and vascular surgery), 50\%, and myeloma, 31\%. Two-year survival on chronic dialysis was higher for patients with parenchymal disease (approximately $80 \%$ ) compared to patients with other ARF etiologies (approximately 50$60 \%)$. The investigators also compared the long-term outcome of ARF patients who developed ESRD after failing to recover renal function with patients who developed ESRD without preceding ARF. For the Australian institution at which this study was performed, survivors of ARF represented 18\% of ESRD patients initiating chronic dialysis. In comparison to non-diabetic ESRD patients without preceding ARF, survival in ARF patients failing to recover renal function was significantly lower over a 5 -year period. Patients in the age range of 50 years and older accounted for this difference.

Several recent studies have evaluated the impact of either dialysis modality or dose on recovery of renal function in ARF. Based on a multicenter trial comparing CRRT and conventional hemodialysis (HD) [41], several lines of evidence suggest a benefit for CRRT with respect to recovery of renal function. First, chronic renal insufficiency at either death or hospital discharge was diagnosed in $17 \%$ of patients whose initial therapy was conventional HD vs. only $4 \%$ of patients whose initial therapy was CRRT (p = 0.01 ). Second, for patients receiving an adequate trial of monotherapy, $92 \%$ of CRRT patients had complete recovery of renal function vs. $59 \%$ of conventional HD ( $p<0.01)$ patients. (An adequate trial was defined as a minimum exposure of $25 \mathrm{~h}$ for CRRT and two treatments of $3 \mathrm{~h}$ or more of duration each for conventional HD.) Finally, a significantly higher percentage of patients crossing over from conventional HD to CRRT had complete recovery of renal function compared to patients crossing over in the opposite direction (45 vs. $7 \%$; $p<0.01)$.

Advances in Acute Renal Failure
Manns et al. [42] assessed the effect of the dialysis modality on recovery of renal function among patients at several institutions in the province of Alberta in Canada. In a sample of 261 patients, recovery of renal function occurred in $80 \%$ of surviving patients whose initial treatment was CRRT, while it occurred in only $63 \%$ of patients whose initial treatment was conventional HD ( $\mathrm{p}=0.06)$. In light of this outcome difference, these investigators also evaluated the cost implications related to recovery vs. non-recovery of renal function after ARF. One analysis focused on costs incurred during the hospitalization in which the ARF episode occurred. This analysis revealed significantly higher costs in patients failing to recover renal function compared to those who recovered renal function (CAD 56,000 vs. 47,700; $\mathrm{p}<0.05$ ). This difference was associated with a significantly longer duration of hospitalization in the former group after the first dialysis treatment ( 47.7 vs. 38.2 days; $p<0.05)$. An additional cost analysis compared annual healthcare costs in the subsequent year after hospital discharge in the same two groups of patients. Largely reflecting the costs of chronic dialysis therapy, a striking sevenfold difference in costs was reported (CAD 73,200 vs. 11,200; $\mathrm{p}<0.05$ ). These data suggest cost comparisons of conventional HD and CRRT that focus on costs related only to supplies and equipment (e.g., fluids, disposables, and machines) without accounting for 'downstream' healthcare costs do not provide a complete picture.

Jacka et al. [43] have also recently assessed the effect of modality on recovery of renal function in ARF. A total of 93 patients, for whom CRRT was the initial modality in 65 and conventional HD the initial modality in 28 , were evaluated during the year 2000. Baseline clinical characteristics of the CRRT group that differed significantly from the conventional HD group included a higher percentage of patients with acute lung injury and liver failure along with higher vasoactive drug requirements. Interestingly, no patients treated initially with conventional HD were changed subsequently to CRRT, while 18 patients treated initially with CRRT were changed subsequently to conventional HD. With respect to patient outcome, unadjusted ICU mortality was significantly higher in the CRRT group but hospital mortality did not differ significantly between the two groups. However, based on multivariate analysis accounting for higher illness severity in the CRRT group at baseline, the renal replacement therapy mode did not have a significant effect on either ICU or hospital mortality. Overall, $32 \%$ of the surviving patients were dialysisdependent at the time of hospital discharge. However, multivariate analysis revealed a striking reduction in the

Blood Purif 2006;24:487-498 


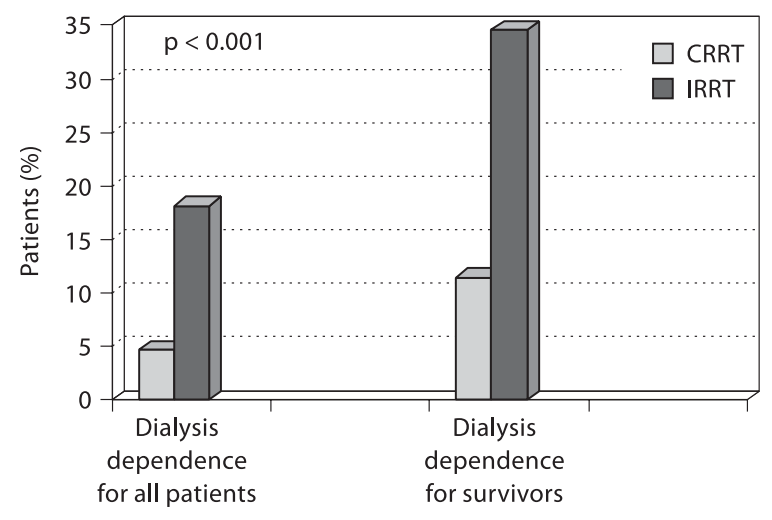

Fig. 3. Dialysis dependence at hospital discharge after ARF ( $\mathrm{n}=$ 1,218 ) according to initial choice of therapy from the BEST Kidney Study. IRRT = Intermittent renal replacement therapy; $\mathrm{CRRT}=$ continuous renal replacement therapy.

likelihood of renal recovery for the conventional $\mathrm{HD}$ group (conventional HD:CRRT odds ratio, 0.04; $\mathrm{p}=$ 0.006) among patients surviving to hospital discharge. In this patient group, 21 of 24 patients (87\%) initially treated with CRRT were free of dialysis dependence at hospital discharge, while only 5 of 14 patients initially treated with conventional HD fell in this category.

Finally, the BEST Kidney investigative group [44] performed an analysis similar to that of Jacka et al. [43]. Of the 1,262 ARF patients who required renal replacement therapy in this study, CRRT was the initial modality in $80 \%$, intermittent renal replacement therapy (IRRT) the initial modality in $17 \%$, and peritoneal dialysis or slow continuous ultrafiltration the initial modality in 3\%. In the IRRT group, although conventional HD was the dominant therapy, intermittent hemofiltration/hemodiafiltration and intermittent SLED/diafiltration also fell in this category. Overall, sepsis/septic shock was the most common clinical diagnosis contributing to the development of ARF, although it was a factor in a significantly higher percentage of patients initially treated with CRRT vs. IRRT ( 50 vs. $38 \%$; $p=0.001$ ). On the other hand, hypovolemia and drug-induced ARF were contributing factors in a significantly higher percentage of patients initially treated with IRRT vs. CRRT ( 29 and 26 vs. 20 and $18 \%$, respectively; $\mathrm{p}=0.005$ and 0.006 , respectively). Overall, $7 \%$ of all patients and $17 \%$ of surviving patients were dialysis-dependent at the time of hospital discharge. However, among patients surviving to hospital discharge, a significantly higher percentage of patients initially treated with IRRT vs. CRRT were dialysis-dependent (35 vs. $11 \%$; $p<0.0001$; fig. 3 ). Likewise, among all patients, dialysis dependence at discharge was significantly more common among patients initially treated with IRRT than CRRT (18 vs. $5 \% ; \mathrm{p}=0.0001$ ).

\section{Conclusions}

In the clinical management of ARF patients in the ICU, many of the advances reported in this review have already been incorporated widely into clinical practice while others await further validation. In much of the world, a CRRT dose of $35 \mathrm{ml} / \mathrm{kg} / \mathrm{h}$ (based on effluent rate) has become the standard of care even as two large randomized trials continue to explore the issue of the effect of CRRT dose on outcome. Moreover, based on informal assessments, the use of the RIFLE criteria for classifying ARF and earlier application of CRRT appear to be occurring more frequently. It is important to emphasize that the vast majority of data supporting these new approaches have come from studies employing convective CRRT.

A final concluding point is that these recent outcome data are largely unique to CRRT and similar data are generally not available for conventional HD and especially SLED, except for evidence that a higher conventional HD dose (in the form of daily HD) improves patient outcome [63]. Specifically, the clinical benefit of convective solute removal noted in several CRRT studies discussed in this review are obviously not relevant to conventional HD and SLED, since these are primarily diffusive therapies. Moreover, the available clinical data suggest a benefit for CRRT over conventional HD with respect to the recovery of renal function while no such data (positive or negative) exist for SLED. In this regard, it is not expected that meaningful outcome data for patients treated with SLED will be available for some time to come due to the relative 'immaturity' of this therapy. On the other hand, the CRRT outcome data presented in this review provide very relevant information to assist clinical decision making for the critically ill ARF patient population. 


\section{References}

1 Lassnigg A, Schmidlin D, Mouhieddine M, et al: Minimal changes in serum creatinine predict prognosis in patients after cardiothoracic surgery: A prospective cohort study. J Am Soc Nephrol 2004;15:1597-1605.

$\checkmark 2$ Loef BG, Epema AH, Smilde TD, et al: Immediate postoperative renal function deterioration in cardiac surgical patients predicts in-hospital mortality and long-term survival. J Am Soc Nephrol 2005;16:195200.

-3 Ryckwaert F, Boccara G, Frappier JM, Colson $\mathrm{PH}$ : Incidence, risk factors, and prognosis of a moderate in creatinine in plasma creatinine after cardiac surgery. Crit Care Med 2002;30:1495-1498.

4 Chertow GM, Burdick E, Honour M, Bonventre JV, Bates DW: Acute kidney injury, mortality, length of stay, and costs in hospitalized patients. J Am Soc Nephrol 2005;16: 3365-3370.

5 Kellum JA, Mehta RL, Angus DC, Palevsky $\mathrm{P}$, Ronco C: The first international consensus conference on continuous renal replacement therapy. Kidney Int 2002;62:18551863.

-6 Kellum JA, Levin N, Bouman C, Lameire N: Developing a consensus classification system for acute renal failure. Curr Opin Crit Care 2002;8:509-514.

$>7$ Bell M, Liljestam E, Granath F, Fryckstedt J, Ekbom A, Martling CR: Optimal follow-up time after continuous renal replacement therapy in acute renal failure patients stratified with the RIFLE criteria. Nephrol Dial Transplant 2005;20:354-360.

-8 Abosaif NY, Tolba YA, Heap M, Russell J, El Nahas AM: The outcome of acute renal failure in the intensive care unit according to RIFLE: Model application, sensitivity, and predictability. Am J Kidney Dis 2005;46: 1038-1048.

-9 Kuitunen A, Vento A, Suojaranta-Ylinen R, Pettila V: Acute renal failure after cardiac surgery: evaluation of the the RIFLE classification. Ann Thorac Surg 2006;81:542-546.

-10 Storck M, Hartl W, Zimmerer E, Inthorn D: Comparison of pump-driven and spontaneous hemofiltration in postoperative acute renal failure. Lancet 1991;337:452-455.

- 11 Ronco C, Bellomo R, Hommel P, Brendolan A, Dan M, Piccinni P, LaGreca G: Effects of different doses in continuous veno-venous hemofiltration on outcomes in acute renal failure: a prospective, randomized trial. Lancet 2000;355:26-30.

12 Clark WR, Ronco C: Renal replacement therapy quantification in acute renal failure: solute removal mechanisms and dose quantification. Kidney Int 1998;53(suppl 66): S133-S137.
13 Clark WR, Turk JE, Kraus MA, Gao D: Dose determinants in continuous renal replacement therapy. Artif Organs 2003;27:815820.

14 Honore PM, Jamez J, Wauthier M, et al: Prospective evaluation of short-term, high-volume isovolemic hemofiltration on the hemodynamic course and outcome in patients with intractable circulatory failure resulting from septic shock. Crit Care Med 2000;28: 3581-3587.

15 Piccinni P, Dan M, Barbacini S, Carraro R, Lieta E, Marafon S, Zamparetti N, Brendolan A, D’Intini V, Tetta C, Bellomo R, Ronco C: Early isovolaemic haemofiltration in oliguric patients with septic shock. Intensive Care Med 2006;32:80-86.

16 Bone RC, Balk RA, Cerra FB, et al: Definitions for sepsis and organ failure and guidelines for the use of innovative therapies in sepsis. The ACCP/SCCM Consensus Conference Committee. Chest 1992;101:16441655.

17 Bellomo R, Ronco C: Indications and criteria for initiating renal replacement therapy in the intensive care unit. Kidney Int Suppl 1998;66:S106-S109.

18 Uchino S, Kellum JA, Bellomo R, et al: Acute renal failure in critically ill patients: a multinational, multicenter study. JAMA 2005; 294:813-818.

19 Metnitz PG, Krenn CG, Steltzer H, et al: Effect of acute renal failure requiring renal replacement therapy on outcome in critically ill patients. Crit Care Med 2002;30:20512058

20 McCarthy JT: Prognosis of patients with acute renal failure in the intensive care unit: a tale of two eras. Mayo Clin Proc 1996;71: 117-126.

21 Hoste EAJ, Lameire NH, Vanholder RC, Benoit DD, DeCruyenaere JMA, Colardyn FA: Acute renal failure in patients with sepsis in a surgical ICU: predictive factors, incidence, comorbidity, and outcome. J Am Soc Nephrol 2003;14:1022-1030.

22 Ympa YP, Sakr Y, Reinhart K, Vincent JL: Has mortality from acute renal failure decreased? A systematic review of the literature. Am J Med 2005;118:827-832.

23 Chertow GM, Levy EM, Hammermeister $\mathrm{KE}$, Grover F, Daley J: Independent association between acute renal failure and mortality following cardiac surgery. Am J Med 1998;104:343-348.

-24 Metnitz PG, Krenn CG, Steltzer H, et al: Effect of acute renal failure requiring renal replacement therapy on outcome in critically ill patients. Crit Care Med 2002;30:20512058

-25 Page B, Viellard-Baron A, Chergui K, et al: Early veno-venous haemofiltration for sepsis-related multiple organ failure. Crit Care 2005;9:R755-R763.
26 Le Gall JR, Lemeshow S, Saulnier F: A new simplified acute physiology score (SAPS II) based on a European/North American multicenter study. JAMA 1993;270:2957-2963.

27 Hoffmann JN, Hartl WH, Deppsich RE, Faist E, Jochum M, Inthorn D: Hemofiltration in human sepsis: evidence for elimination of immunomodulary substances. Kidney Int 1995;48:1563-1570.

$>28$ Kellum JA, Johnson JP, Kramer D, Palevsky P, Brady JJ, Pinsky MR: Diffusive vs. convective therapy: effects on mediators of inflammation in patients with severe systemic inflammatory response syndrome. Crit Care Med 1998;26:1995-2000.

29 Joannes-Boyau O, Rapaport S, Bazin R, Fleureau $C$, Janvier $G$ : Impact of high volume hemofiltration in hemodynamic disturbance and outcome during septic shock. ASAIO J 2004;50:102-109.

30 Cole L, Bellomo R, Journois D, Davenport P, Baldwin I, Tipping P: High volume hemofiltration in human septic shock. Intensive Care Med 2001;27:278-286.

-31 Cole L, Bellomo R, Hart G, Journois D, Davenport $\mathrm{P}$, Tipping $\mathrm{P}$, Ronco C: A phase II randomized controlled trial of continuous hemofiltration in sepsis. Crit Care Med 2002; 30:100-106.

32 Brendolan A, D’Intini V, Ricci Z, et al: Pulse high-volume hemofiltration. Int J Artif Organs 2004;27:398-403.

33 Ratanarat R, Brendolan A, Piccinni P, et al: Pulse high-volume hemofiltration for treatment of severe sepsis: effects on hemodynamics and survival. Crit Care 2005;9: R294-R302.

34 Ziegler EJ, Fisher CJ, Sprung CL, et al: Treatment of gram-negative bacteremia and septic shock with HA-1A monoclonal antibody against endotoxin. N Engl J Med 1991;324: 429-436.

\$35 Fisher CJ, Agnosti J, Opal S, et al: Treatment of septic shock with the tumor necrosis factor: Fc fusion protein. N Engl J Med 1996; 334:1697-1702.

36 Bernard GR, Vincent JL, Laterre PF, et al: Efficacy and safety of recombinant human activated protein $\mathrm{C}$ for severe sepsis. $\mathrm{N}$ Engl J Med 2001;344:699-709.

37 Clark WR, Leypoldt JK, Henderson LW, Mueller BA, Scott MK, Vonesh EF: Quantifying the effect of changes in the hemodialysis prescription on effective solute removal with a mathematical model. J Am Soc Nephrol 1999;10:601-610.

38 Clark WR, Ronco C: CRRT efficiency and efficacy in relation to solute size. Kidney Int Suppl 1999;72:S3-S7.

-39 Kaplan AA: Therapeutic plasma exchange for the treatment of rapidly progressive glomerulonephritis. Ther Apher 1997;1:255259. 
-40 Bouman C, Oudemans-VanStraaten H, Tijssen J, et al: Effects of early high-volume continuous veno-venous hemofiltration on survival and recovery of renal function in intensive care patients with acute renal failure: a prospective, randomized trial. Crit Care Med 2002;30:2205-2211.

-41 Mehta RL, McDonald B, Gabbai F, et al: A randomized clinical trial of continuous vs. intermittent dialysis for acute renal failure. Kidney Int 2001;60:1154-1163.

42 Manns B, Doig CJ, Lee H, et al: Cost of acute renal failure requiring dialysis in the intensive care unit: clinical and resource implications of renal recovery. Crit Care Med 2003; 31:449-455.

43 Jacka MJ, Ivancinova X, Gibney N: Continuous renal replacement therapy improves renal recovery from acute renal failure. Can J Anesth 2005;52:327-332.

44 BEST Kidney: Choice of initial renal replacement therapy for acute renal failure in ICU: impact on survival and renal recovery (manuscript under review).

45 Brunet S, Leblanc M, Geadah D, Parent D, Corteau S, Cardinal J: Diffusive and convective solute clearance during continuous renal replacement therapy at various dialysate and ultrafiltration flow rates. Am J Kidney Dis 1999;34:486-492.

$\checkmark 46$ Huang Z, Gao D, Clark WR: Convective renal replacement therapies for acute renal failure and end-stage renal disease. Hemodial Int 2004;8:386-393.

-47 Liao Z, Zhang W, Poh CK, Huang Z, Hardy PA, Kraus MA, Clark WR, Gao D: Kinetic comparison of different acute dialysis therapies. Artif Organs 2003;27:802-807.
48 Casino FG, Marshall MR: Simple and accurate quantification of dialysis in acute renal failure patients during either urea nonsteady state or treatment with irregular or continuous schedules. Nephrol Dial Transplant 2004;19:1454-1466.

49 Kielstein JT, Kretschmer U, Ernst T, et al: Efficacy and cardiovascular tolerability of extended dialysis in critically ill patients: a randomized controlled study. Am J Kidney Dis 2004;43:342-349.

50 Ricci Z, Ronco C, Bachetoni A, et al: Solute removal during continuous renal replacement therapy in critically ill patients: convection vs. diffusion. Crit Care 2006;10: R67.

51 Clark WR, Mueller BA, Kraus MA, Macias WL: Extracorporeal therapy requirements for patients with acute renal failure. J Am Soc Nephrol 1997;8:804-812.

52 Tremblay R, Ethier J, Querin S, Beroniade V, Falardeau P, Leblanc M: Veno-venous continuous renal replacement therapy for burned patients with acute renal failure. Burns 2000;26:638-643.

53 Leblanc M, Garred L, Cardinal J, Pichette V, Nolin L, Quimet D, Geadah D: Catabolism in critical illness: estimation from urea nitrogen appearance and creatinine production during continuous renal replacement therapy. Am J Kidney Dis 1998;32:444-453.

54 Gettings LG, Reynolds HN, Scalea T: Outcome in post-traumatic acute renal failure when continuous renal replacement therapy is applied early vs. late. Intensive Care Med 1999;25:805-813.
55 Clark WR, Mueller BA, Alaka KJ, Macias WL: A comparison of metabolic control by continuous and intermittent therapies in acute renal failure. J Am Soc Nephrol 1994; 4:1413-1420.

56 Bent P, Tan HK, Bellomo R, et al: Early and intensive continuous hemofiltration for severe acute renal failure after cardiac surgery. Ann Thorac Surg 2001;71:832-837.

57 Liano F, Gallego A, Pascual J, et al: Prognosis of acute tubular necrosis: an extended prospectively contrasted study. Nephron 1993; 63:21-31.

58 Elahi M, Lim MY, Joseph RN, Dhannapuneni RRV, Spyt TJ: Early hemofiltration improves survival in post-cardiotomy patients with acute renal failure. Eur J Cardiothor Surg 2004;26:1027-1031.

59 Sugahara S, Suzuki H: Early start on continuous hemodialysis therapy improves survival rate in patients with acute renal failure following coronary bypass surgery. Hemodialysis Int 2004;8:320-325.

60 Mehta RL, Pascual MT, Soroko S, Chertow GM: Diuretics, mortality, and nonrecovery of renal function in acute renal failure. JAMA 2002;288:2547-2553.

61 Uchino S, Doig GS, Bellomo R: Diuretics and mortality in acute renal failure. Crit Care Med 2004;32:1669-1677.

62 Bhandari S, Turney JH: Survivors of acute renal failure who do not recover renal function. Q J Med 1996;89:415-421.

63 Schiffl H, Lang SM, Fischer R: Daily hemodialysis and the outcome of acute renal failure. N Engl J Med 2002;346:305-310. 